PAPER

\title{
Cognitive changes after epilepsy surgery in the posterior cortex
}

\author{
R Luerding, F Boesebeck, A Ebner
}

J Neurol Neurosurg Psychiatry 2004;75:583-587. doi: 10.1136/jnnp.2003.014746

See end of article for authors' affiliations ......................

Correspondence to: $R$ Luerding, Department of Neurology, University of Regensburg,

Universitätsstraße 84, D-93053 Regensburg, Germany; ralf.luerding@ medbo.de

Received 14 March 2003

In revised form

22 August 2003

Accepted

1 September 2003 Objective: The relationship between the posterior cortex and cognitive functions is still a relatively open
field. There are no studies on populations in which functions of posterior structures were examined by a standardised neuropsychological examination before and after posterior resections. Changes in cognitive performance are regularly observed after epilepsy surgery in the temporal lobe. However, information about neuropsychological impairments after resections in the posterior cortex is poor, owing to the relatively low proportion of cortical resections in this area.

Methods: We retrospectively studied changes in cognition in the neuropsychological data of 28 patients prior to and 6 months after posterior cortical resections.

Results: Cognition significantly showed differences in performance intelligence quotient compared with verbal intelligence quotient. Post-operative verbal intelligence consistently increased, whereas performance intelligence decreased. There was no effect regarding the lesion side, continuation of seizures, or reduction of visual field after surgery. Epilepsy surgery in this area did not lead to significant differences in general intelligence after surgery.

Conclusion: Functions of posterior areas could be described by standardised neuropsychological measures. Posterior regions contribute to explicit attentional and visuoconstructional abilities. Epilepsy surgery in the posterior cortex bears no risk for substantial decline in general cognition although some discrete impairment in performance intelligence may occur.
$\mathrm{N}$ europsychological examination prior to and after cortical resection for the treatment of lesional epilepsies is regularly performed in the pre-surgical diagnostic phase. In temporal lobe epilepsies, the neuropsychological data have been shown to contribute to the localisation of the epileptogenic zone. ${ }^{12}$ Furthermore, the data enable a prognosis for the post-surgical cognitive outcome. $^{3}$ As most operations in epilepsy surgery programmes are carried out in temporal and frontal areas, information about the neuropsychological changes after posterior cortical resections is inadequate because the cognitive outcome in this group has not been investigated systematically.

Many different functions are subserved by posterior brain regions; this contributes to the complexity of evaluating changes after surgery. ${ }^{4-6}$ The parietal lobe, like the frontal structures, performs higher level processes. Difficulties in visual, visuospatial orientation, and in visuoperceptive tasks in patients with parietal lobe epilepsy have been reported. ${ }^{7}$

The parietal lobe integrates sensation and cognition. Inferior parietal lobe function is not purely perceptional, but acts as a sensorimotor interface. ${ }^{4}$ Parietal lobe functions are further described as a link of sensation to action. ${ }^{5}$ The parietal lobe serves as a pivot point for synthesizing multiple modalities into a common perception. Using functional magnetic resonance imaging, parietal lobes are strongly activated every time an object is the target of action. The parietal lobes prepare representations of objects for action. ${ }^{6}$ Reliable measurements of changes in cognitive function changes based upon quantitative neuropsychological measurement after a substantial loss of parietal lobe parenchyma have not yet been reported.

The main function of the occipital cortex is perception, integration, and processing of visual perceptions. The primary visual cortex is mainly located in the medial aspect of the occipital cortex (areas Vl and dV3). Supplementary visual areas are located in the temporo-occipital border, with the lingual gyrus harbouring area V2 and VP and the fusiform gyrus harbouring area $\mathrm{V} 4 .^{8}$ Two accessory visual pathways from the occipitally located visual cortex to parietal (dorsal route) and temporal (ventral route) structures have been described. ${ }^{9}$ The dorsal route is thought to be responsible for the visual control of movements, ${ }^{10}$ whereas the ventral route may be associated with the "what" content of visual information. ${ }^{11}$ Lesions involving the occipital visual cortex are regularly associated with visual field defects contralateral to the lesion side. A minor reduced visual field is often undetected by the patients as a result of small nystagmus movements virtually "filling" the visual gaps. ${ }^{8}$ Colour blindness has been reported after lesions involving $V 4^{12}$ and loss of movement detection following lesions in the V5 area. ${ }^{13}$ Furthermore, reports of certain forms of visual agnosia, prosopagnosia, and alexia after bilateral lesions in the lateral occipital lobe and in the temporo-occipital border have been published. $^{14} 15$

Because of the manifoldness of parietal and occipital functions, there is a need for a methodological study to obtain a precise prognosis of cognition changes after a lesionectomy in the posterior cortex. The aim of this study was to explore cognition changes in 28 patients after epilepsy surgery in the posterior cortex. We focused on cognitive and perceptual abilities typically represented by parietal, occipital, and temporo-occipital structures.

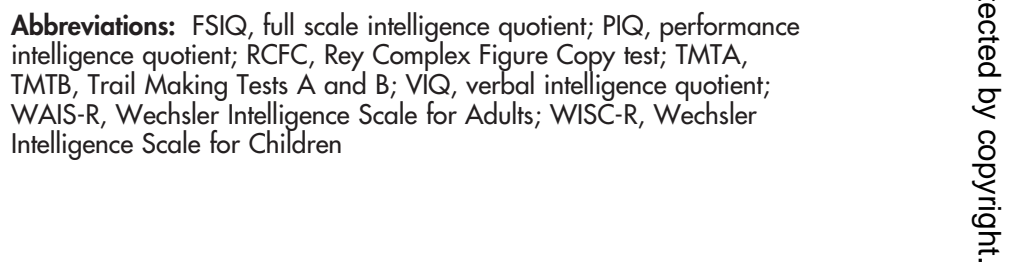




\section{PATIENTS AND METHODS}

\section{Patients}

We selected 28 patients (16 male) with lesional epilepsies in the posterior cortex from the epilepsy surgery databank of the Bethel Epilepsy Center (Bielefeld, Germany). Detailed data for each patient are shown in table 1 .

Patients without pre- or post-operative neuropsychological data were excluded from the study. Mental retardation was not a criterion for exclusion. Mean age at surgery was 27.8 years (range 13-53). The epileptogenic lesions consisted of five parietal, five occipital, two parieto-occipital, 14 temporo-occipital, and two temporo-parieto-occipital (19 right). Pre-surgical evaluation included video EEG monitoring with superficial scalp EEG for localisation of the epileptogenic focus for all patients. Supplemental invasive EEG using subdural grids, epidural electrodes, or depth electrodes was applied in 14 cases. EEG data and ictal semiology were analysed for possible predictive factors for post-operative seizure outcome. Results of this analysis are reported elsewhere. ${ }^{16}$ Magnetic resonance imaging using $T_{1}$ and $T_{2}$ weighted pulse sequences was available for every patient. In patients with lesions in the left hemisphere, speech dominance was tested by the Wada test ${ }^{17}$ in five cases and/or cortical stimulation in seven. Twenty three patients were right handed, three were left handed, and two were ambidextrous. From nine patients with left sided lesions, eight were right handed. The one left handed patient showed right sided language lateralisation, and one right handed patient showed bilateral language lateralisation. From 19 patients with right sided lesions, 14 were right handed, two were left handed, and two were ambidextrous. The left handed patient and seven right handed patients showed all left language lateralisation. All patients underwent epilepsy surgery between 1991 and 2000. The histological diagnosis was vascular malformations (cavernomas and angiomas) in five patients, low grade tumours in nine, malformations of cortical development in two, and gliosis in twelve.

\section{Neuropsychological tests}

The visual field was tested prior to and 6 months after operation using a Goldmann perimeter. Patients without reduction in visual field related to the surgery were categorised as constant as were those with unchanged preoperatively existing reduced visual field. The visual field was categorised as reduced in patients whose cortical resection led to a new or enlarged visual field defect.

All patients underwent a comprehensive neuropsychological evaluation described in detail elsewhere. ${ }^{18}$ Both the evaluation and the post-surgical examination were performed weeks apart from the video EEG monitoring in order to ensure measurement in a phase of steady medication and in a seizure free interval.

Verbal and nonverbal cognition was measured using a short form of the German version of the Wechsler Intelligence Scale for Adults Revised (WAIS-R), the Hamburg-Wechsler Intelligenztest für Erwachsene (Revision 1991). ${ }^{19}$ Verbal intelligence quotient (VIQ) was measured with the subtests (a) general information, (b) comprehension, and (c) similarities. The measure of performance intelligence quotient (PIQ) consisted of the subtests (a) block design, $(b)$ digit symbol test, and $(c)$ picture completion. Patients aged below 16 years were assessed with the homologous subscales from the German version of the Wechsler Intelligence Scale for Children Revised (WISC-R), the Hamburg Wechsler Intelligenztest für Kinder Revision 1983. ${ }^{20}$

\section{Statistical methods}

Means of pre-surgically assessed VIQ were compared with post-surgically assessed VIQ. Means of PIQ measured before

\begin{tabular}{|c|c|c|c|c|c|c|c|c|}
\hline No & $\begin{array}{l}\text { Age } \\
\text { (years) }\end{array}$ & Sex & $\begin{array}{l}\text { Lesion } \\
\text { side }\end{array}$ & $\begin{array}{l}\text { Resected } \\
\text { regions }\end{array}$ & Hand & Wada* & Histology & Pre-op FSIQ \\
\hline 1 & 30 & $M$ & Left & $P$ & Left & Right & Gliosis & 94 \\
\hline 2 & 14 & $\mathrm{~F}$ & Right & $P$ & Right & Left & Gangloglioma & 53 \\
\hline 3 & 36 & $M$ & Right & $P$ & Right & Left & Dnt & 128 \\
\hline 4 & 16 & $M$ & Left & $P$ & Right & & Oligodendroglioma & 77 \\
\hline 5 & 33 & $M$ & Right & $\mathrm{P}$ & Right & & Gliosis & 38 \\
\hline 6 & 37 & $\mathrm{~F}$ & Right & 0 & Right & & Gangloglioma & 97 \\
\hline 7 & 20 & $M$ & Right & 0 & Right & & Gliosis & 91 \\
\hline 8 & 34 & $M$ & Right & 0 & Right & & Gliosis & 112 \\
\hline 9 & 38 & $M$ & Left & 0 & Right & & Gliosis & 93 \\
\hline 10 & 30 & $\mathrm{~F}$ & Right & 0 & Left & & Gliosis & 81 \\
\hline 11 & 30 & $M$ & Right & $\mathrm{PO}$ & Left & Left & Astrocytoma $\|^{\circ}$ & 47 \\
\hline 12 & 17 & M & Left & $\mathrm{PO}$ & Right & & Gliosis & 62 \\
\hline 13 & 13 & $\mathrm{~F}$ & Right & TO & Right & & Gliosis & 89 \\
\hline 14 & 25 & $M$ & Left & TO & Right & Left & Angioma & 108 \\
\hline 15 & 18 & $\mathrm{~F}$ & Right & TO & Right & & Oligodendroglioma & 83 \\
\hline 16 & 24 & $M$ & Right & TO & Right & & Mcd & 132 \\
\hline 17 & 37 & $\mathrm{~F}$ & Right & TO & Right & Left & Gliosis & 90 \\
\hline 18 & 34 & $M$ & Right & TO & Right & Left & Gliosis & 114 \\
\hline 19 & 42 & M & Left & TO & Right & Bilateral & Gliosis & 63 \\
\hline 20 & 17 & $\mathrm{~F}$ & Right & TO & Right & Left & Dnt & 119 \\
\hline 21 & 20 & $\mathrm{~F}$ & Left & TO & Right & Right & Gangloglioma & 56 \\
\hline 22 & 18 & $M$ & Right & TO & Right & Left & Mcd & 84 \\
\hline 23 & 28 & $\mathrm{~F}$ & Right & TO & Ambi & & Angioma & 64 \\
\hline 24 & 23 & $\mathrm{~F}$ & Left & TO & Right & Right & Dnt & 57 \\
\hline 25 & 30 & $\mathrm{~F}$ & Right & TO & Right & Left & Cavernoma & 78 \\
\hline 26 & 31 & $M$ & Leff & TO & Right & & Angioma & 115 \\
\hline 27 & 19 & M & Right & TPO & Right & & Gliosis & 115 \\
\hline 28 & 53 & $\mathrm{~F}$ & Right & TPO & Ambi & & Angioma & 47 \\
\hline \multicolumn{9}{|c|}{$\begin{array}{l}\text { *Wada, language lateralisation by Wada test } \\
\text { P, parietal; O, occipital; TO, temporo-occipital; TPO, temporo-parieto-occipital; MCD, malformation of cortical } \\
\text { development; DNT, dysembryoblastic neuroepithelial tumour; FSIQ, full scale IQ; hand, handedness; ambi, } \\
\text { ambidextrous; pre-op, pre-operative. }\end{array}$} \\
\hline
\end{tabular}


surgery were compared with those after surgery in $t$ tests for repeated measures.

A multivariate analysis of variance with repeated measures was used to analyse differences between VIQ and PIQ in different groups. Each one of the repeated measurements consisted of the variables VIQ and PIQ. Intra-subject factor was the measurement of VIQ and PIQ before and after surgery. Group membership (right $v$ left sided surgery, continuation of seizures $v$ freedom from seizures after surgery, and reduced $v$ constant visual field after surgery) served as inter-subject measures. Homogeneity of variance was tested prior to the multivariate analysis of variance with repeated measures.

Univariate analysis of variance was computed to test for the intra-subject effect of differences in the variance of VIQ and PIQ in pre- and post-surgical assessments in each group. Univariate analysis of variance was also performed to analyse the inter-subject effects in differences of variances in preand post-surgical VIQ and PIQ in each group.

\section{RESULTS}

\section{General seizure outcome}

Twelve patients $(43 \%)$ were free of seizures 2 years after surgery (Engel classification Ia and Ib), and another 11 patients $(39 \%)$ had a worthwhile seizure reduction (Engel Ic to III). For an analysis of post-surgical interictal epileptiform discharges see Boesebeck et al). ${ }^{16}$

\section{Visual impairments related to surgery}

Half of the patients $(n=14)$ had a reduced visual field after surgery, with six having mild impairments (five upper and one lower square anopia) and eight suffering from hemianopia.

\section{Pre-operative IQ}

Mean full IQ (WAIS-R) was 85.3, ranging from 38 to 132 with a mean pre-operative VIQ of 89.0 and a mean PIQ of 84.3.

\section{Comparison of pre- and post-operative intelligence} There was no significant effect in a $t$ test for repeated measures comparing the means of pre- with post-surgical VIQ (89.04 and 91.11 respectively, $\mathrm{t}=-1.03, \mathrm{p}=0.314$ ). There was also no significant difference between pre- and post-operative PIQ ( 84.25 and 80.64 respectively, $t=1.32$, $\mathrm{p}=0.199$ ). The post-surgical outcome of VIQ showed an ascending tendency, whereas post-surgical outcome of PIQ showed a descending tendency.

In multivariate testing there were significant effects in the intra-subject factor pre- and post-operative measure in each of the inter-subject "lesion side" groups $(\mathrm{df}=2,28, \mathrm{~F}=3.54$, $\mathrm{p}<0.05)$, continuation of seizures $(\mathrm{df}=2,28, \mathrm{~F}=3.56$, $\mathrm{p}<0.05)$, and reduction of visual field $(\mathrm{df}=2,28, \mathrm{~F}=3.86$, $\mathrm{p}<0.05$ ). Post-operative measures differed significantly from pre-operative measures in each group.

There was no significant difference between subjects with right or left sided lesions, between subjects with or without continuation of seizures, or between subjects with or without a reduction of visual field. In univariate tests there were also no effects between subjects with right or left sided lesions, between subjects with or without continuation of seizures, or between subjects with or without a reduction of visual field. Lesion side, continuation of seizures, or reduction of visual field did not affect the difference of variances of PIQ and VIQ. The significant difference of pre- and post-surgical measure was equal in each group.

VIQ tended to increase after surgery whereas PIQ tended to decrease. Comparing the mean post-operative with the mean pre-operative VIQ, post-operative improvement did not reach significance. Comparing the mean post-operative with the mean pre-operative PIQ, post-operative reduction did not reach significance either, but the directions of the variances of VIQ and PIQ were significantly different within subjects using the multivariate analysis of variance with repeated measures, substantiating a loss of PIQ compared with VIQ.

Using univariate testing, the intra-subject factor measured for VIQ or PIQ was not significant pre-operatively, but was significant post-operatively for both VIQ and PIQ in all groups, regardless of whether the lesion was in the left or right hemisphere $(\mathrm{df}=1,28, \mathrm{~F}=7.19, \mathrm{p}<0.05)$, whether seizures continued or not $(\mathrm{df}=1,28, \mathrm{~F}=7.30, \mathrm{p}<0.05)$, and whether visual field was diminished or not $(\mathrm{df}=1,28$, $\mathrm{F}=7.91, \mathrm{p}<0.01$ ). There were significant differences between post-surgical PIQ and VIQ, but not between pre-surgical PIQ and VIQ, again regardless of side of surgery, continuation or not of seizures, or reduction or not of visual field. The postsurgical difference was equal in each group. Before surgery, patients showed no significant difference between PIQ and VIQ, thus the significant differences between them found after surgery must be an effect of the surgery. PIQ failed to show a variance in the same direction as VIQ; PIQ was reduced compared with VIQ. Lesion side, reduction or not of visual field after surgery, and continuation or not of seizures after surgery had no effect on the variation of cognitive outcome (figs 1-3).

To exclude a pure visuoperceptive effect on the difference of VIQ and PIQ, the post-surgical outcome of other tests with visuoperceptual components were compared with the subtests of PIQ. Z scores of subtests obtained in the pre-surgical assessment were compared with those measured in the postoperative examination. Negative z scores resulted when postoperative cognition was lower than pre-operative cognition, positive $\mathrm{z}$ scores show an increase in cognition after surgery (fig 4).

In comparison with other visuoperceptive and visuoconstructive measures, all subtests of PIQ from the WAIS-R and Trail Making Test A (TMTA), a task of visual search and psychomotor speed, showed a descending tendency after surgery. Trail Making Test B (TMTB) and copying the Rey Complex Figure (RCFC), measures of frontal lobe performance, ${ }^{21} 22$ showed an ascending tendency. A $\mathrm{Z}$ value

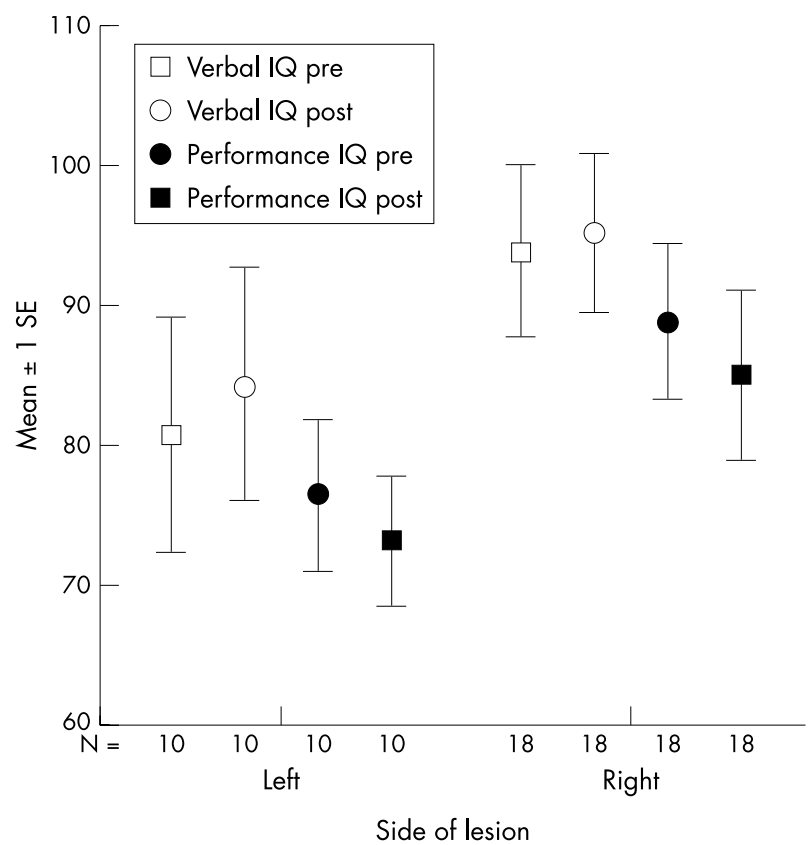

Figure 1 A significant difference in $P I Q$ compared with $\mathrm{VIQ}$ in patients with right or left hemisphere surgery. 


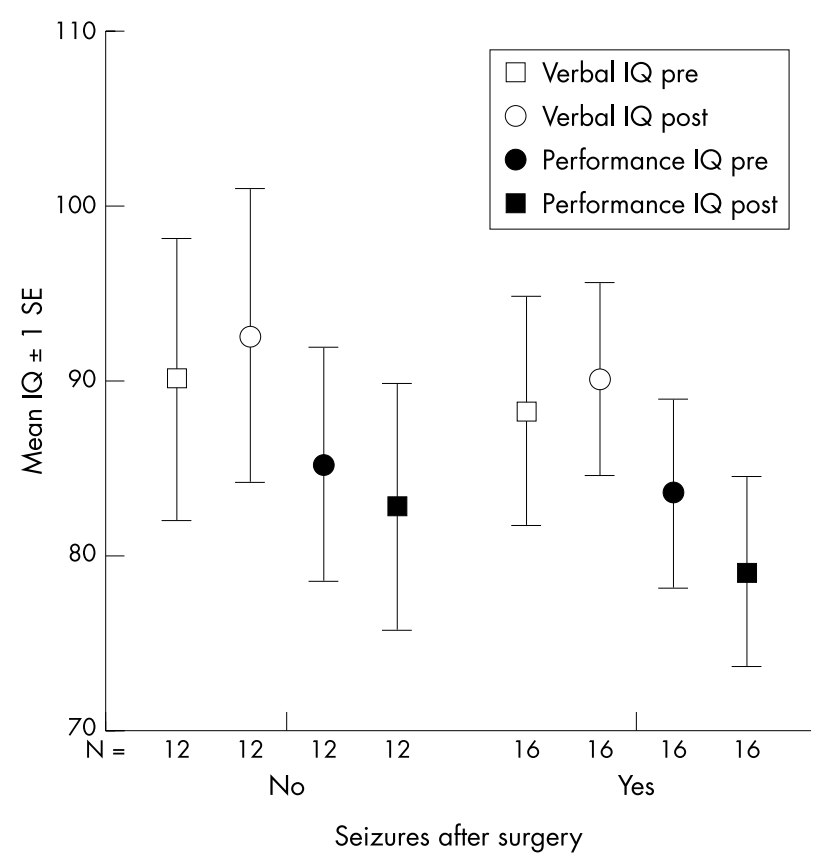

Figure 2 A significant difference in $P I Q$ compared with $V I Q$ in patients with or without seizures after surgery.

difference $<1$ is not considered significant, so a difference of a single subtest does not reach significance. Visuoperceptive abilities are required to finish subtests of PIQ, TMTB, and RCFC. However, the results of these visuoperceptive measures showed a quite different variability. A purely visuoperceptive disability after surgery cannot explain the effect of lower PIQ. The only explanation for these differences in visuoperceptive measures is a change in specific cognitive abilities.

\section{DISCUSSION}

The results of our study show that posterior cortical resections, either in the dominant or non-dominant hemisphere, do not lead to significant changes in general intelligence. PIQ changed in a significantly different direction from VIQ regardless of lesion side. Because 23 patients (82\%) had a worthwhile seizure reduction or were seizure free, an effect of a new pattern of seizure related cognitive impairment is unlikely. A change in VIQ could be expected after left sided parietal surgery, but in fact was not found. One reason may be that preoperative diagnostics such as Wada and invasive evaluation were sufficiently precise to avoid damage during surgery to any of the language areas of the brain and thus prevent any deterioration of the patient's language skills. Alternatively, the VIQ subtests in WAIS-R may have limited sensitivity for measurement of language perception. Additionally, a higher risk of diminished PIQ after right sided surgery could be expected, but the lesion side had no significant effect. Higher functions of posterior regions do not seem to be lateralised.

This is in contrast to the outcome after temporal lobe resections. Several authors have shown a significant increase in full scale IQ (FSIQ) after non-dominant temporal lobe resection and only a slight increase or a decrease in FSIQ after dominant temporal lobe resections. ${ }^{3}$ An increase in PIQ and VIQ in seizure free patients and no change when seizures continued has been reported. ${ }^{23}$ There was no neuropsychological change in patients with mesial temporal lobe epilepsy, but a significant decline in patients with lateral temporal lobe

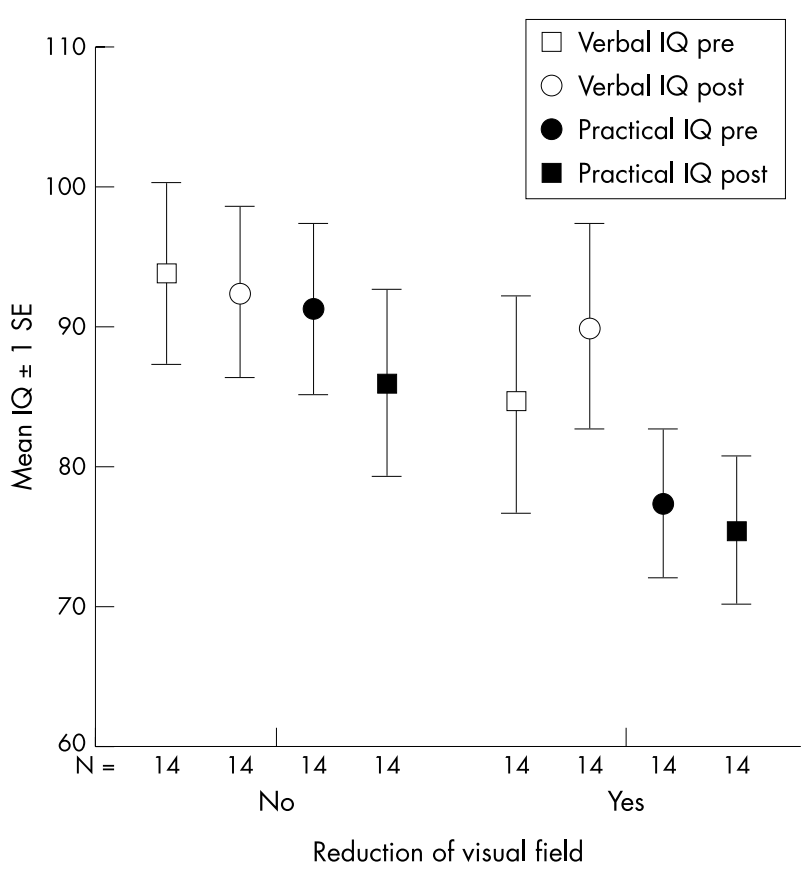

Figure 3 A significant difference in PIQ compared with $\mathrm{VIQ}$ in patients with or without reduced visual field after surgery.

epilepsy. ${ }^{24}$ PIQ was almost invariably shown to be improved compared to baseline IQ, regardless of the side of temporal resections. ${ }^{25}{ }^{26}$ The effect of dominant temporal lobe resections on VIQ seems to be somewhat inconsistent, with either decreasing ${ }^{24}$ or increasing scores, whereas right temporal lobe resection regularly leads to increasing scores. ${ }^{24}$ In contrast to this, our data showed a mild decrease of PIQ compared with VIQ after epilepsy surgery in the posterior cortex, which was significant for the differences of direction of variances for both VIQ and PIQ. This effect was independent of post-operative visual field defects and seizure outcome.

For interpretation, it is necessary to consider the neuropsychological characteristics of the subtests applied in the evaluation of PIQ. A review ${ }^{22}$ of PET and lesion studies on subtests is summarised as follows. The subtest "digit symbol"

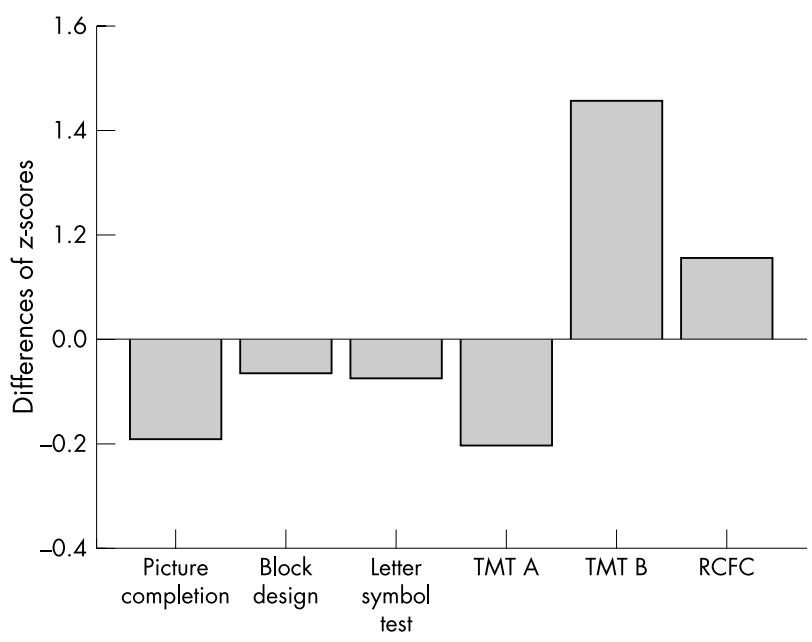

Figure 4 Differences of $z$ scores in subtests picture completion, block design, and letter symbol test from WAIS-R in comparison with the visuoperceptive and visuoconstructive measures Trail Making Tests $A$ and B (TMTA and TMTB), and Rey Complex Figure Copy (RCFC) test. 
is sensitive for even minimal brain damage. Efficiency in digit symbol is affected regardless of the lesion side. PET studies show a bilateral increase in posterior regions of glucose metabolism while working on this task, with a predominance for the right hemisphere. The subtest "picture completion" does not differentiate between lesion side. Efficiency in picture completion is relatively unaffected after diffuse brain damage. Bilateral metabolic increases are most prominent in right posterior hemisphere but are also seen in the left parietal lobe. The third subtest of WAIS-R included in our analysis was "block design". Efficiency in block design is likely to be affected least by left hemisphere lesion, except when the left parietal lobe is involved. Each hemisphere contributes to the processing of this task. Examining glucose metabolism in subjects working on the block design test showed an increasing activation in posteriorparietal areas, especially in the right hemisphere.

Although neuropsychological data were collected retrospectively from the standardised neuropsychological examination with tests not assembled prospectively to measure posterior functions, the applied tests are sensitive for detecting posterior functionality.

Difficulties in visual, visual spatial orientation, and visual perceptive tasks in patients with parietal lobe epilepsy have been published. ${ }^{7}$ There are descriptions of parietal lobe functions based upon qualitative evaluation after lesions and on functional imaging. Parietal lobe functions serve as a link of sensation to action. ${ }^{5}$ The parietal lobes are strongly activated when an object is the target of action and they prepare representations of objects for action. ${ }^{6}$ Inferior parietal lobe function is not purely perceptional but is a function of a sensorimotor interface. ${ }^{4}$ Lesions of the parietal lobe are associated with an impairment of object recognition, constructional apraxia, and attention. ${ }^{4}$

Our results are congruent with descriptions of parietal lobe functions in lesion studies and in functional imaging studies. A survey of the literature points to a complexity of parietal lobe functions, which can be partially described by the results in our study.

\section{CONCLUSION}

Even complex functionality of the parietal lobes can be examined with standardised neuropsychological tests when functions are examined in an isolated manner, such as the measurement of a pre/post difference after epilepsy surgery in the posterior cortex.

Our study revealed no risk for substantial impairment of general cognition after epilepsy surgery in the posterior cortex. However, a minor decrease of PIQ may appear in some patients, which has to be taken into account when considering epilepsy surgery. Furthermore, the data underline the great importance of suitable neuropsychological test methods covering the particular functions of the cortical areas being considered for resection.

\section{Authors' affiliations}

R Luerding, Department of Neurology, University of Regensburg, Universitätsstraße 84, D-93053 Regensburg, Germany

F Boesebeck, Von Bodelschwinghsche Anstalten Bethel, Clinic Gilead IV, D-33164 Bielefeld, Germany
A Ebner, Epilepsy Centre Bethel, Clinic Mara I, Epilepsy Surgery Program, D-33164 Bielefeld, Germany

Competing interests: none declared

\section{REFERENCES}

1 Jones-Gotman M. Presurgical neuropsychological evaluation for localization and lateralization of seizure focus. In: Luders $\mathrm{H}$, ed. Epilepsy surgery. New York: Raven Press, 1991:469-75.

2 Kneebone AC. Presurgical neuropsychological evaluation for localization and lateralization of the epileptogenic zone. In: Luders H, Comair G, eds. Epilepsy surgery, 2nd edn. Philadelphia: Lippincott Williams \& Wilkins, 2001:469-75.

3 Chelune GJ. Using neuropsychological data to forecast postsurgical cognitive outcome. In: Luders H, ed. Epilepsy surgery. New York: Raven Press, 1991:477-85.

4 Freund $\mathrm{HJ}$. The parietal lobe as a sensorimotor interface: a perspective from clinical and neuroimaging data. Neuroimage 2001;14:142-6.

5 Freund HJ. Sensorimotor processing in parietal neocortex. Adv Neurol 2000;84:63-74.

6 Buccino G, Binkofski F, Fink GR, et al. Action observation activates premotor and parietal areas in a somatotopic manner: an fMRI study. Eur J Neurosci $2001 ; 13: 400-4$.

7 Siegel AM, Williamson PD. Parietal lobe epilepsy. Adv Neurol 2000:84:189-99.

8 Kolb B, Whishaw IQ. Der Occipitallappen (The occipital lobe). In: Neuropsychologie (German edn). Heidelberg, Berlin, Oxford: Spektrum Akademischer Verlag GmbH, 1996:204-22.

9 Ungerleider LG, Mishkin M. Two cortical visual systems. In: Ingle DJ, Goodale MA, eds. Mansfield analysis of visual behaviour. Cambridge: MIT Press, 1982

10 Milner AD, Goodale MA. Visual pathways to perception and action. Prog Brain Res 1993;95:317-37.

11 Ungerleider LG, Haxby JV. What and where in the human brain. Curr Opin Neurobiol 1994:4:15-165.

12 Sacks O, Wassermann R. The case of the colorblind painter. NY Rev Books 1987;34:25-33.

13 Zihl J, von Cramon D, Mai N. Selective disturbance of movement vision after bilateral brain damage. Brain 1983;106:313-40.

14 Goodale MA, Milner AD, Jakobson LS, et al. A neurological dissociation between perceiving objects and grasping them. Nature 1991;349:154-6.

15 Brazis PW, Masdeu JC, Biller J. The localization of lesions affecting the cerebral hemispheres. In: Localization in clinical neurology, 3rd edn. Boston: Little, Brown \& Co, 1996:449-533.

16 Boesebeck F, Schulz R, May T, et al. Lateralizing semiology predicts the seizure outcome after epilepsy surgery in the posterior cortex. Brain 2002; 125:2320-31.

17 Wada J, Rasmussen T. Intracarotid injection of sodium amytal for the lateralization of cerebral speech dominance: experimental and clinical observations. J Neurosurg 1960;17:266-82.

18 Jokeit H, Seitz RJ, Markowitsch HJ, et al. Prefrontal asymmetric interictal glucose hypometabolism and cognitive impairment in patients with temporal lobe epilepsy. Brain, 1997 Dec, 120:2283-94.

19 Tewes U. Handbuch und Testanweisung für den Hamburg-Wechsler Intelligenztest für Erwachsene, Revision 1991. Bern, Göttingen, Toronto, Seattle: Hans Huber, 1994.

20 Tewes U. Hamburg-Wechsler-Intelligenztest für Kinder-Revision 1983: HAWIK-R. Handbuch und Testanweisung. Bern Stuttgart Wien: Hans Huber, 1984.

21 Ettlin T, Kischka U. Bedside frontal lobe testing. The frontal lobe score. In: Miller BL, Cummings JL, eds. The human frontal lobes. New York, Guilford Press, 1999:233-46.

22 Lezak MD. Neuropsychological assessment. New York: Oxford University Press, 1995.

23 Wachi M, Tomikawa M, Fukuda $M$, et al. Neuropsychological changes after surgical treatment for temporal lobe epilepsy. Epilepsia 2001:42(Suppl 6):4-8.

24 Seidenberg M, Hermann B, Wyler AR, et al. Neuropsychological outcome following anterior temporal lobectomy in patients with and without the syndrome of mesial temporal lobe epilepsy. Neuropsychology 1998; 12:303-16.

25 Blackmoore CB, Falconer MA. Long-term effect of anterior temporal lobectomy on certain cognitive functions. J Neurol Neurosurg Psychiatry 1967;30:364-7.

26 Rausch R, Crandall PH. Psychological status related to surgical control of temporal lobe seizures. Epilepsia 1982;23:191-202.

27 Ivnik RJ, Sharbrough FW, Laws ER. Anterior temporal lobectomy for control of partial complex seizures: information for counseling patients. Mayo Clin Proc 1988;63:783-91. 